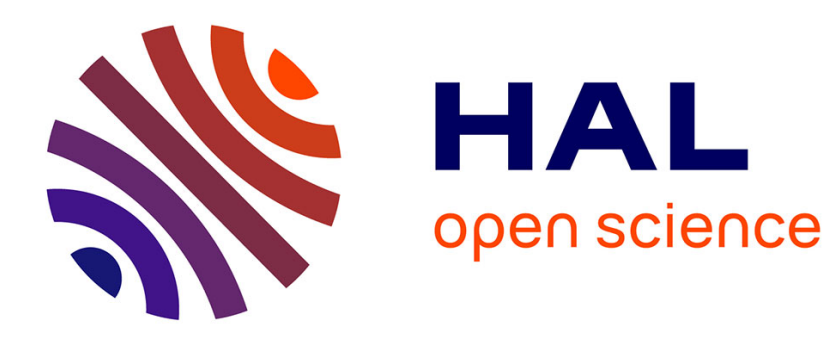

\title{
Strain nonuniformities and plastic instabilities
}

L.P. Kubin, Y. Estrin

\section{To cite this version:}

L.P. Kubin, Y. Estrin. Strain nonuniformities and plastic instabilities. Revue de Physique Appliquée, 1988, 23 (4), pp.573-583. 10.1051/rphysap:01988002304057300 . jpa-00245805

\section{HAL Id: jpa-00245805 https://hal.science/jpa-00245805}

Submitted on 1 Jan 1988

HAL is a multi-disciplinary open access archive for the deposit and dissemination of scientific research documents, whether they are published or not. The documents may come from teaching and research institutions in France or abroad, or from public or private research centers.
L'archive ouverte pluridisciplinaire HAL, est destinée au dépôt et à la diffusion de documents scientifiques de niveau recherche, publiés ou non, émanant des établissements d'enseignement et de recherche français ou étrangers, des laboratoires publics ou privés. 


\title{
Strain nonuniformities and plastic instabilities
}

\author{
L.P. Kubin and Y. Estrin*
}

Laboratoire de Métallurgie Physique. Université de Poitiers, UA CNRS 131, 40, Avenue du Recteur Pineau, 86022 Poitiers Cedex, France

* Technische Universität Hamburg-Harburg, Harburger Schlosstr. 20, 2100 Hamburg 90, F.R.G.

(Reçu le 26 mai 1987, révisé le 24 septembre 1987, accepté le 5 octobre 1987)

\begin{abstract}
Résumé. - L'auto-organisation des structures de dislocations, des lignes ou bandes de glissement, les instabilités plastiques macroscopiques, constituent, à différentes échelles d'observation, des manifestations typiques d'un comportement coopératif non linéaire. Cet aspect général est discuté sous l'angle des relations entre les propriétés dynamiques des dislocations, leur comportement collectif, le comportement plastique local du matériau étudié et les conditions d'apparition d'une déformation nonuniforme. Il en résulte une classification des instabilités en deux principales catégories qui sont illustrées par deux exemples particuliers: les bandes de Lüders (instabilités de type $h$ ) et l'effet Portevin-Le Chatelier (instabilités de type 5 ).

Abstract. - Self-organization of dislocation structures, slip line and band patterning, and macroscopic plastic instabilities are typical manifestations, at different scales of observation, of nonlinear cooperative phenomena. This general aspect is discussed with emphasis on the relations between the dynamic properties of dislocations, their collective behavior, the local plastic properties of a material and the conditions for the onset of nonuniform plastic flow. Instabilities can be classified into two main categories which are illustrated by two particular examples: Lüders bands (instabilities of the $h$ type) and the Portevin-Le Chatelier effect (instabilities of the $S$ type).
\end{abstract}

\section{Introduction and physical background.}

Strain nonuniformities and plastic instabilities (SNPI) are of obvious practical interest since their occurrence can damage both the forming properties and lifetimes in service of metallurgical products. They also are of great theoretical interest because they touch a difficult and still largely unsolved problem of the connection between defect properties and the bulk deformation behavior. In particular, one would like to know more about the reasons for which a cooperative behavior of large densities of dislocations can set in within a sizeable portion of the gauge length of a deforming specimen.

In what follows, we limit ourselves to SNPI in ductile crystalline materials, in conditions of uniaxial straining, and to mechanisms which can be understood in terms of dislocation properties. Severe types of localization, such as necking or shear banding are discussed by Needleman in this volume.

For simplicity we reduce the problem to one dimension, $x$, parallel to specimen axis. Strain localization occurs, by definition, when the strain rate locally accelerates. This amounts to a spontaneous breaking of the translational symmetry along $x$.
A formal approach consists in considering first that plastic flow is uniform: $\partial \varepsilon / \partial x \equiv 0$, where $\varepsilon$ denotes the strain. The onset of nonuniformity, then, indicates that a solution $\varepsilon=\varepsilon(t)$ of some hypothetical constitutive equation loses its stability, leading to solutions of the form $\varepsilon=\varepsilon(x, t)$. This suggests that the concepts of bifurcations ( 1 ), from uniform to nonuniform behavior, and of stability analysis of physically founded constitutive equations are potentially well adapted tools for handling the problem.

slip lines and bands are the most common type of strain localization (cf. $(2,3,4)$ and $\S 2)$; their existence proves, indeed, that plastic flow is inherently nonuniform, although most models which describe it implicitly assume the opposite to be true. More generally, a prerequisite for obtaining some knowledge about the spatial distribution of strain rates within a deforming specimen is that, somehow, position variables be introduced into constitutive equations. This was attempted only recently, as classical plasticity has up to now been more concerned with temporal than with spatial aspects.

Plastic instabilities of ten manifest themselves by anomalies in the deformation curves. They can 
induce strain bursts in deformation with constant stress rate, inverted creep curves $\lg \dot{\varepsilon}$ vs. $\varepsilon$, a decrease in the load carrying capacity of the specimen in deformation with constant strain rate (5). More generally, we characterize plastic instabilities by the fact that the total average strain rate, $\bar{\varepsilon}$, defined as

$$
\overline{\dot{\varepsilon}}=(1 / L) \int_{0}^{L} \dot{\dot{\varepsilon}}(x, t) d x,
$$

where $L$ is the gauge length, exceeds its prescribed value in uniform deformation. Plastic instabilities are necessarily associated with strain localization, as shown in $\S 2$, but the reverse is not necessarily true. The formation of slip lines does not usually affect the value of $\overline{\dot{\varepsilon}}$ to the point that deformation curves take irregular shapes.

From this qualitative discussion we draw two conclusions: (i) plastic instabilities cannot be understood in macroscopic terms only. In particular, it should be kept in mind that all quantitities extracted from deformation curves, such as strain hardening. rates, strain rates, sometimes strain rate sensitivities, are global averages (cf. eq. (1)) over nonuniformly strained elements rather than intrinsic material properties. (ii) The examination of microstructural mechanisms at mesoscale (i.e. at intermediate, light microscopy scale), combined with the definition of straining conditions and taking into account local compatibility requirements, yields in principle all the informations needed for a complete understanding of plastic instabilities.

Mesoscale properties are, in their turn, dictated by collective dislocation effects. The organization of dislocation populations in time and space represents at microscale a problem equivalent to that of strain localization at mesoscale. Here again, there exists a uniform solution to the equations describing the distribution of dislocation densities within a deforming specimen. Instability of this solution leads to patterning, i.e. to the occurrence of spatially organized structures (see Kratochvil, this volume).

Expressions derived, within some approximation, from Orowan's law,

$$
\dot{\varepsilon}=\rho_{m} b v,
$$

provide the necessary connection between micro and mesoscale ( $\rho_{m}$ : density of mobile dislocations, of average velocity $v$, b: modulus of the active Burgers vector). In nonuniform conditions, this expression must be understood as being valid at local level ( $x$ fixed) only. A geometrical factor of the order of unity has been dropped in eq. (2).

To sum up, understanding SNPI in physical terms necessitates the consideration of three different scales of organization (micro, meso, and macro), connected by averaging or integration steps, such as eqs. (1) and (2). Spatial and/or temporal patterning is present at each scale and can formally be treated as stemming from the instability of uniform plastic flow, provided that appropriate time and length scales are introduced. A last question arises: what is the physical reason causing such kind of particular collective behavior?

The reason is nonlinarity, i.e. the property that the differential equations which determine the evolution of local strain rates or of dislocation populations have no general solution which is a linear combination of particular solutions. In that sense, SNPI belong to a very large class of phenomena, of prominent importance in physics, chemistry, biology..., for which one examines how a system brought far from thermodynamic equilibrium responds to uniform excitations by selforganization. Plasticity is intrinsically an irreversible phenomenon and to elaborate on it, it could seem natural to make use of irreversible thermodynamics (6). Such approach does not seem able to produce consistent results which realistically incorporate defect properties (7), however. The investigations outlined in $\S 2$ are of purely dynamic character. Although they have not reached full achievement, they have already produced promising results. A classification of instabilities, which recovers earlier elaborations, is further derived, and in $\$ 3$ we go into a more detailed examination of two particular cases: Lüders type instabilities and the Portevin-Le Chatelier effect.

There exists a considerable bulk of literature on SNPI. In what follows we only sketch a few essential concepts, without going into heavy formalism, and we focus on the understanding of general trends, rather than on the compilation of particular aspects.

We conclude this section by emphasizing the analogy between SNPI and other non linear collective phenomena. Fig. 1, reproduced from the work of Müller (8), shows an example of nonuniform chemical reactions (chemical waves) where activity is confined to the vicinity of mobile spiral fronts. There is an obvious analogy between individual molecules and dislocations (microscale), chemical waves and strain nonuniformities (mesoscale), average concentrations and deformation curves (macroscale). The modelling of chemical waves involves solving a set of nonlinear differential equations describing the rate of each reaction, the diffusion of each species, conservation conditions, and boundary effects. We now examine the analogous ingredients needed to study SNPI.

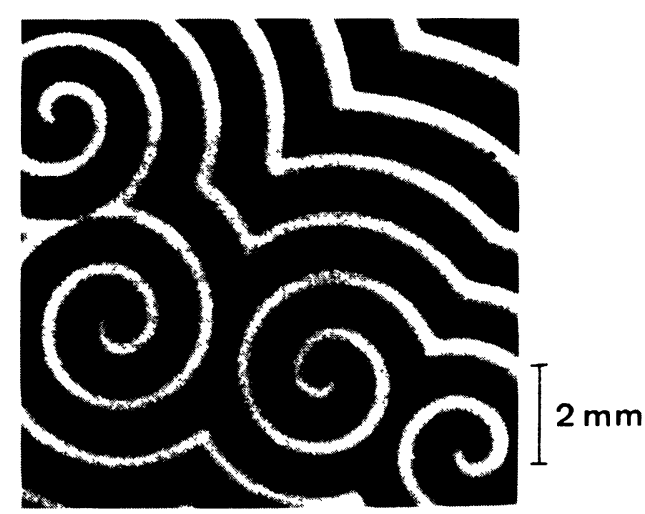

Fig. 1. - Chemical waves, after Müller. Reproduced from ref. (8), by courtesy of Martinus Nijhoff.

\section{The dynamic approaches to SNPI.}

According to the methods outlined in $\S 1$, the investigation of SNPI amounts to solving a set of coupled nonlinear differential equations whose ingredients pertain to two or three different scales and involve time and space as evolutionary variables. It is required that bifurcatjon conditions be determined, which is not too difficult, and that solutions beyond the bifurcation point be obtained, which is usually less simple. 
Understandably, there exists no general solution to this problem and even when dealing with specific cases, simplifying assumptions, sometimes very rough ones, have to be made. Since the main objective is to reproduce the main features of some well-characterized types of unstable behavior, the crucial point consist in properly introducing the nonlinear terms and couplings by whicis the dynamics will be governed. Once this is correctly done, the results obtained are not very sensitive to details of the individual mechanisms and this justifies some roughness of a model, which can be kept simple.

In this section, we review a few basic ingredients which can be used at macro, meso and micro scales, stressing the various levels of approximation and recalling some recent advances.

2.1. - MECHANICAL CONSTRAINTS. One must first assume that conditions of uniaxial straining remain valid throughout the specimen. This is admissible when localizations are not too severe, as is the case for those considered here, and especially near the stability limits. Then, stresses and strains can be taken in the forms $\sigma(x, t)$ and $\varepsilon(x, t)$, respectively.

A first constraint fixes deformation conditions. These are trivial in uniform, but not in nonuniform deformation. The average (total) plastic strain rate is given by eq. (1), and a similar form holds for the total elastic strain rate, $\bar{\varepsilon}_{\mathrm{el}}$ :

$$
\dot{\varepsilon}_{e l}=(1 / L) \int_{0}^{L}(\dot{\sigma}(x, t) / E) d x,
$$

where $E$ is the Young modulus. Thus, deformation at constant total applied strain rate, ' $\dot{\varepsilon}_{a}$, is expressed in the form:

$$
\dot{\varepsilon}_{\mathrm{a}}=\dot{\varepsilon}_{\mathrm{el}, \mathrm{s}}+\dot{\bar{\varepsilon}}_{\mathrm{el}}+\overline{\dot{\varepsilon}}
$$

where $\dot{\epsilon}_{e l, s}$ is the elastic deformation of the testing system. Other modes of deformation (creep, constant stress rate, of cyclic deformation) are dealt with in a similar manner.

A second constraint arises from stress equilibrium or from inertial effects. After a fast local instability event, stresses get equilibrated along the specimen at the velocity $c$ of acoustic waves, i.e. it takes a time $t_{e}=L / c \simeq 10^{-5}$ s to obtain stress equilibrium (see (9) for a discussion). This value is to be compared with the typical time scale associated with the instability, $\Delta t_{i}$. Dynamic effects will prevail if, for instance, a local strain of $\Delta \varepsilon=10^{-2}$ is achieved with a local average strain rate of $\dot{\varepsilon}=\Delta \varepsilon / \Delta t_{i} \simeq 10^{3} \mathrm{~s}^{-1}$. Such fast events are met with mechanical twinning, sometimes with the Portevin-Le Chatelier effect, or with local dislocation avalanches $(10,11)$. In such cases one must take care to avoid setting the tensile system in vibration at its eigenfrequencies (see (4) for more detail) and to ensure that inertial effects do not affect too much the recorded stress vs. time behavior.

The application of the fundamental equation of dynamics to an element of volume subject to a stress gradient yields $(12,13)$ :

$$
\partial \sigma / \partial x=m \partial^{2} u / \partial t^{2}
$$

where $m$ is the mass density and $u$ the displacement. This expression can further be reexpressed in terms of stress and strain rate by taking into account the deformation conditions.

When stress equilibrium is assumed, as is usually the case, it suffices to express the condition that the load $P=\sigma A$ ( $A$ : cross-sectional area of the specimen) is uniform. Under constant load, we locally have: $d \ln \sigma / d \varepsilon= \pm d \ln A / d \varepsilon$, where the upper sign refers to compression and the lower one to tension. Assuming that there is no volume change during plastic deformation, $d \ln A=-d \varepsilon$, where $\varepsilon$ has here a meaning of the absolute value of strain. Combining these two expressions, we obtain:

$$
d \sigma=\mp \sigma d \varepsilon,
$$

and equivalent expressions for other modes of deformation. In summary, mechanical constraints pose two conditions, one of type (4) and one of type (5).

2.2. - DISLOCATION POPULATIONS. Referring to the example of chemical waves given above, we see that ingredients at inicroscale will be more complicated for dislocations than for inolecules: dislocation glide is assisted by temperature and also by stress, climb and cross-slip must sonetines be taken into account, and above all, the dislocation densities are not constant because of multiplication.

The aim of the studies reported below is to examine the evolution with time of dislocation densities, and to determine characteristic length scales for their structural organization.

In a first step we consider time dependence only and split the total dislocation density into a mobile density, $\rho_{m}$, and an almost immobile one, $\rho_{f}$. Denoting by $\dot{\rho}_{k}^{+}(k=m$ or $f)$ and $\dot{p}_{k}$ the rates of creation and of annihilation of the corresponding species, respectively, we can write down the kinetic balance equations of the form:

$$
\mathrm{d} \rho_{\mathrm{k}} / \mathrm{dt}=\dot{\rho}_{\mathrm{k}}^{+}+\dot{\rho}_{\mathrm{k}} \cdot
$$

In practice, a set of two (or several) coupled non linear differential equations is obtained (14-17). The terms at right-hand side can be modelled in several different ways (18). For example, a term proportional to - $\rho_{m}^{2}$ accounts for the mutual annihilation of two mobile segments. A term proportional to $-\rho_{m} \rho_{f}^{2}$ used in (18) describes the trapping of a mobile dislocation by a sessile dipole and contributes both to $\dot{\rho}_{f}^{+}$(with a negative sign) and to $\dot{p}_{m}^{-}$(with a positive sign). It introduces both a strong nonlinearity and a coupling between the two densities.

The stress or strain rate dependence of the various elementary mechanisms, in particular multiplication (the term in $\dot{\rho}_{m}^{+}$) and recovery (the term in $\dot{\rho}_{\vec{F}}^{-}$) can be incorporated through simple phenomenological forms in the coefficients which determine their relative weight. The flow stress, or an effective stress obtained by subtracting from it an athermal contribution proportional to $\rho_{F}^{1 / 2}$, usually enters as a control parameter in the multiplication term. Alternatively, strain, rather than time, can be chosen as the evolutionary variable (14). Fig. 2 reproduces a typical strain dependence of the mobile and forest densities obtained within such a frame. We notice that at sinall strains the increase in the mobile density is steeper. This has 


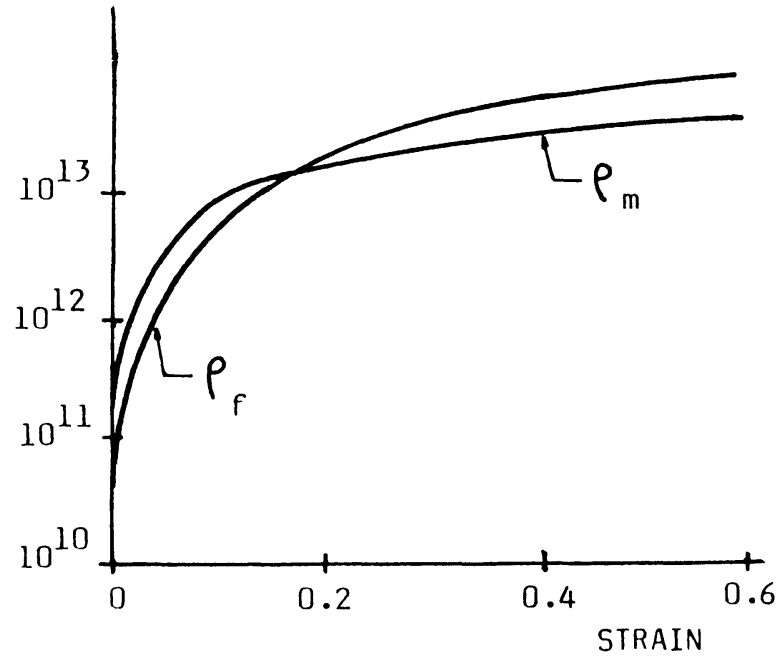

Fig.2. - A typical strain dependence of the mobile $\left(\rho_{r n}\right)$ and forest $\left(\rho_{f}\right)$ dislocation densities $(14)$.

important consequences regarding the local values of strain hardening rate at the very beginning of plastic flow (cf. § 2.3). For large strains, the two densities saturate to values $P_{m s}$, and $P_{f s}$ given by the conditions that $\mathrm{d} \rho_{k} / \mathrm{dt}$ vanishes in eqs. (6), and it can be noticed that the mobile density saturates first. This behavior can be understood by considering that mobile dislocations must be created before being trapped, so that the evolution of the forest density is always shifted in time (strain) with respect to that of the mobile density.

Ananthakrishna and coworkers (15-17) made use of a similar scheme to investigate the Portevin-Le Chatelier (PLC) effect. In their work, the total density is split into several populations which interact via a set of chemical-like reactions, of which we reproduce three particular ones:

$$
\begin{gathered}
m \rightarrow m+m \\
m+m \longrightarrow f+f \\
m+f \rightarrow 0
\end{gathered}
$$

The first reaction describes the multiplication of mobile dislocations, at a rate determined by a breeding constant and an average velocity, which for simplicity is taken constant during a creep experiment. The two other reactions describe the mutual trapping of two mobile dislocations and the annihilation of a mobile dislocation with a dislocation of the forest. Stress-strain curves are then computed by expressing the differential forms for the concentrations/densities of each species, selecting reasonable values for the rate constants and inserting the strain rate via eq. (2). In the case of the PLC effect, where a particular interplay between two mobile populations (fast and slow) must be introduced (cf. $\$ 3.3$ ), the stepped deformation curve reproduced in fig. 3 is obtained (compare with fig. 10).

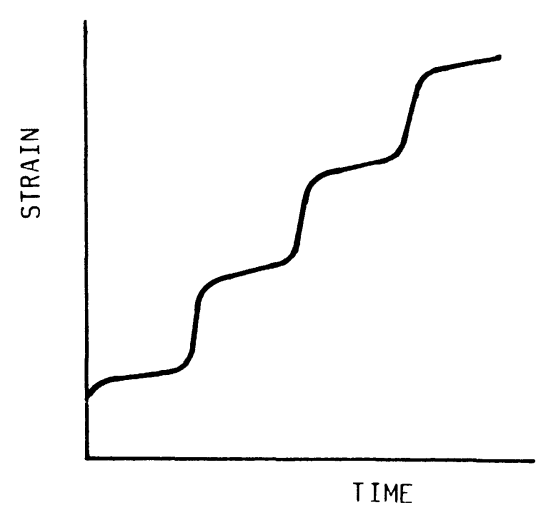

Fig. 3. - A stepped deformation curve obtained by simulating the evolution of dislocation populations during the PLC effect. After Ananthakrishna and Sahoo (15).

This investigation illustrates a constant feature in such type of modelling of plastic instabilities. The cooperative nonlinear behavior dominates the dynamics to the point that a fully detailed knowledge of individual dislocation mechanisms is not really needed. This is probably the reason why materials differ much more in their individual dislocation properties than in their overall mechanical properties.

In the elaborations discussed above, only temporal aspects of instabilities were accounted for. The question of the origin and prediction of dislocation patterning is a long-standing, somewhat controversial, problem in plasticity (cf. (19), and (20) for a brief review). In short, it seems very difficult to consistently take into account the mutual interactions between all the dislocations present in the microstructure. Both energy minimization criteria and dynamic self-organization effects can be thought of as determining the characteristic dimensions of cells, cell-walls, and other organized structures.

Two directions of investigation have recently been explored: one makes use of a reaction-diffusion scheme to model dislocation patterning. The balance equations for dislocation populations, then, contain diffusion-like terms, from which length scales for the organization of dislocation structures can be determined (cf. (13), (2l) and Kratochvil, this volume). Within this frame Brechet (13) has examined the system of equations obtained by coupling eqs. (4) and (5-1) with a simplified reaction-diffusion equation. Several types of SNPI were examined (precipitate shearing, instabilities in fatique, Lüders front propagation and the PLC effect). The solutions obtained reproduce at least qualitatively the main spatial and temporal aspects involved in these four types of instabilities. Fatigue patterning in f.c.c. crystals was investigated by Walgraef and Aifantis (21) who obtained, as a result, a bifurcation diagram where successive bifurcations lead to the formation of a matrix structure and further of Persistent slip Bands. In that case also the length scales characteristic of the organization of dislocation structures can be estimated.

In another approach, dislocation patterning is numerically simulated using a procedure which 
derives from cellular automata techniques (19). Some results of such "computer experiments" pertaining to the propagation of Lüders fronts are reported in $\S 3.2$ (see also Lépinoux, this volume).

2.3. - PHENOMENOLOGY. Elaborations at mesoscale may serve to integrate the collective aspects of dislocation properties into simple material properties, valid at local scale, such as the strain hardening rate (SHR), $h$, and the strain rate sensitivity (SRS), S. SNPI can also be modelled at this phenomenological scale, by attributing to these two coefficients some prescribed dependence on e.g. stress, strain rate or strain.

We illustrate such elaborations by two simplified examples. In more rigorous treatments, care must be taken in justifying the constitutive equations used.

Several different types of differential constitutive equations are commonly used, for instance

$$
d \sigma=h d \varepsilon+S d l n \dot{\varepsilon},
$$

or

$$
\mathrm{dln} \sigma=\gamma \mathrm{d} \varepsilon+\nu \mathrm{dln} \dot{\varepsilon} .
$$

Here, $h=(\partial \sigma / \partial \varepsilon)_{\dot{\varepsilon}}$, and $S=(\partial \sigma / \partial \ln \dot{\varepsilon})_{\varepsilon}$, with $\nu=5 / \sigma$ and $\gamma=h / \sigma$. These material parameters can vary during straining, and in case of strain nonuniformity, their values can also be nonuniform.

To illustrate the concept of linear stability analysis, we consider a hypothetical uniform deformation curve obtained by combining eq. (7-1) with conditions of straining at constant strain rate (eq. (4), taken in uniform conditions) and assuming stress equilibrium. We examine the response of this solution to a local fluctuation in slip activity (i.e. a small strain fluctuation within the specimen), which locally introduces small increments in stress, strain, and strain rate, $\delta \sigma, \delta \varepsilon$, and $\delta \dot{\varepsilon}$, respectively. Such mesoscale fluctuations globally affect a few slip planes during a small time interval, so that the applied load can be considered constant, and therefore they must be connected through eqs $(5-2)$ and $(7-1)$, hut not through eq. (4), since the deformation system cannot control them. Eliminating $\delta \sigma$ by combining eqs. $(5-2)$ and $(7-1)$, we obtain:

$$
0=(h \pm \sigma) \delta \varepsilon+S(\delta \dot{\varepsilon} / \dot{\varepsilon}) \text {, }
$$

and

$$
\delta \dot{\varepsilon} / \delta \varepsilon=-(1 / \dot{\varepsilon})(h \pm \sigma) / S=\lambda \text {. }
$$

$\lambda$ represents the acceleration of strain (9), but linear stability analysis shows that it is also the bifurcation parameter (14). When $\lambda$ is negative, i.e. when deformation decelerates, any local fluctuation in strain is bound to exponentially die out with time since the section considered will be further subject to strain rates smaller than the other parts of the specimen. Then, the uniform solution is stable. When $\lambda$ is positive, i.e. when deformation accelerates, the strain rate exponentially increases within the considered section (provided that $\lambda$ remains constant). The uniform solution is, therefore, unstable, being destabilized by any fluctuation present within the specimen. Once a fluctuation has started growing, leading to some sizeable strain nonuniformity, the deformation system can, possibly, control it. Then, either growth will continue unbounded, leading to rupture, or the nonuniformity will eventually saturate. The condition $\lambda=0$, then, is equivalent to an instability criterion.

In this simplified derivation the spatial extent of fluctuations is not considered, since the constitutive equation used does not contain the position variable $x$. As a consequence, nothing can be said about the length scales of the nonuniformities.

It follows from this discussion that if both the SRS and the SHR are positive, we have a stable situation $(\lambda<0)$.

If the SRS is positive and if $(h \pm \sigma)$ gets negative, we have an instability of type $h$, as pictured in fig. 4 (a).

If the SRS gets negative, the SHR being positive, a more violent type of instability is obtained, because $\lambda$ changes sign by going through infinite values, cf. eq. (9) and fig. 4 (b). Since $\lambda$ is infinite, a jump in the strain rate will occur at constant strain. The most representative example of such type $S$ instability is the PLC effect.
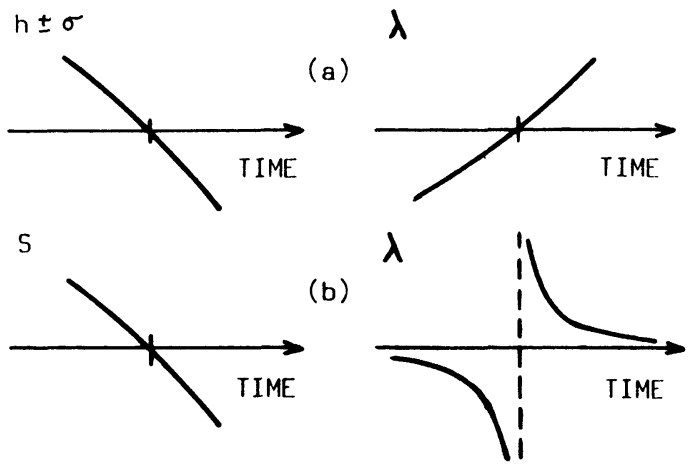

Fig. 4. - Instabilities of type $h(a)$ and of type $S$ (b). $\lambda$ is the bifurcation coefficient representing the acceleration of strain. Uniform plastic deformation is unstable when $\lambda$ is positive.

In a second example, we examine a particular form of the constitutive relation (7-1), which can be obtained by developing Orowan's law (eq. (2)) as follows:

$$
\dot{\varepsilon} \propto \rho_{\mathrm{m}}(\varepsilon) b \exp \left[\left(\sigma-\alpha G b \rho_{f}^{1 / 2}(\varepsilon)\right) / s\right] .
$$

Here we have assumed that the dislocation densities are unique functions of strain. The exponential form for the velocity derives from a first-order expansion in the exponential of the Arrhenius law, and the effective stress is obtained by subtracting from the flow stress an "athermal" contribution proportional to $\rho_{f}^{1 / 2}(\alpha$ is a constant and $G$ is the shear modulus). $S$ is easily identified with the SRS : $S=(\partial \sigma / \partial \ln \varepsilon)_{\varepsilon}$.

Using the definition of the strain hardening rate, $h=(\partial \sigma / \partial \varepsilon)_{\dot{\varepsilon}}$, we have:

$$
h=-s\left(\partial \ln \rho_{m} / d \varepsilon\right)_{\dot{\varepsilon}}+\alpha G b\left(\partial \rho_{f}^{1 / 2} / \partial \varepsilon\right)_{\dot{\varepsilon}} \text {. }
$$

The evolution of the local strain hardening rate under increasing strain can be inferred from fig. 2 , where the evolution of the dislocation densities is depicted. Its strain dependence is represented on fig. 5. One can notice that this variation strongly differs, especially at small strains, from the average behavior recorded on deformation curves. At small strains, the negative 
contribution to $h$ arising from the multiplication of mobile dislocations always prevails (14). The softening effect of the initial rate of production of mobile dislocations cannot be compensated by forest hardening, at least during the very first stages of plastic flow. The SHR is negative and

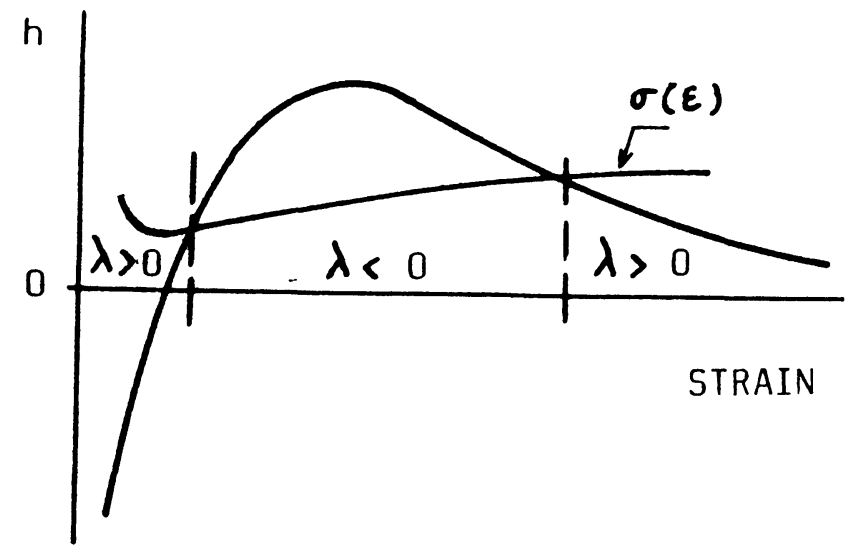

Fig. 5. - Schematic strain dependence of the local strain hardening rate. The two unstable regions $(\lambda>0)$ correspond to the formation of slip lines and bands at small strains and to the onset of necking in tension at large strains.

deformation must, then, be nonuniform. We interpret this behavior as the cause of the existence of slip lines and slip bands. Indeed, at the beginning of plastic flow the strain rate accelerates, going from almost zero to some prescribed value. As discussed above, uniform deformation is unstable in such conditions. Such instability is necessarily bounded because, with increasing strain, forest hardening builds up, while the mobile dislocation density saturates first (cf. fig. 2). As soon as $h$ becomes positive, uniform deformation becomes stable, i.e. deformation tends to uniformize. Slip is activated in new areas of the specimen, to compensate for the loss of activity in saturated regions. This spreading of slip activity can take place either in an orderly (Lüders band-like) manner or by random initiation of slip lines and bands along the specimen.

At large strains, the forest density also saturates, and the (then positive) strain hardening coefficient decreases with increasing strain. In tension, the condition $(h-\sigma)=0$ is met for a certain value of the strain, and according to eq. (9) another instability of type $h$ sets in (cf. fig. 5). Indeed, what is recovered here is nothing else than the familiar Considere criterion for the onset of necking. We notice from eq. (9) that the initial rate of development of the neck will be strongly influenced by the value of the (positive) SRS.

Finally, fig. 6 reproduces a typical time dependence of the local strain and strain rate in creep conditions. The strain rate goes through a maximum when slip is activated and further slows down, while the strain saturates (14). This behavior is in agreement with experimental measurements of the growth of slip steps performed by Neuhäuser and coworkers on single crystals of copper-base alloys $(2,3,4)$.

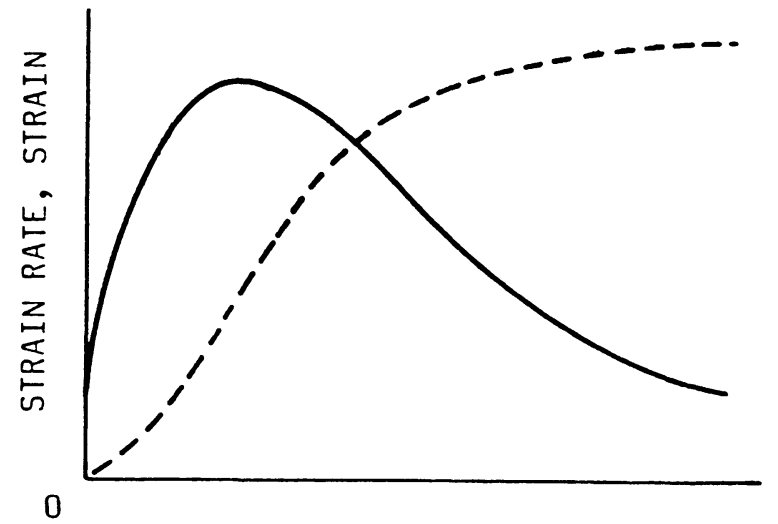

TIME

Fig. 6. - Variation of the local creep rate $\dot{\varepsilon}$ with time (schematic). Dashed: the corresponding strain vs time curve. After (14).

3. Lüders bands and the Portevin-Le Chatelier effect.

3.1. - INTRODUCTION. Assuming that plastic flow is isothermal, we have defined two types of instabilities, those of type $h$ and those of type $s$. The PLC effect is the only well-documented example of type $S$ instability. There are indications that mechanical twinning is also of type $S$ (22), but the microstructural mechanisms which may, in that case, lead to a negative microscopic SRS associated with twin nucleation are not understood.

By contrast, there are many different instabilities of type $h$ and four subgroups can be distinguished. Multiplication softening is associated with a rapid increase in mobile dislocation density and gives rise to yield drops, accompanied by the formation of slip lines and bands which may be propagating (Lüders bands). Structural softening is observed when the microstructure becomes more penetrable to dislocations during straining, or when the SHR attains sufficiently low values. A particular case occurs when the microstructure is destroyed by the mobile dislocations; this leads to the formation of dislocation-free channels (see e.g. (4) for more detail). Instabilities of the matrix structure in fatigue result in two types of SNPI, the formation of Persistent Slip Bands (23) and Neumann's instabilities (24). Finally geometrical instabilities can be caused by slip plane rotation in single crystals or by textural softening in polycrystals, terminating in necking.

In what follows we examine in more detail Lüders bands and fronts and the PLC effect. Only a qualitative description is given here and more detail will be found in $(25-27)$ and in (9).

These two propagative instabilities were first discovered and examined in the course of early investigations of plasticity, in $18 \overline{36}$ (28) and 1860 (29) (Lüders bands in mild steels) and in 1909 (30) and further in 1924 (31) (the PLC effect). The socalled blue-brittleness of steels related to the PLC effect has been noticed by Adamson even earlier, in 1878 (see quotation in (32)). Since then, these two phenomena have been observed in many b.c.c. and f.c.c. solution hardened alloys, but, despite a considerable amount of experimental investigation, no consistent quantitative treatment is available. This is because in both cases the connection between micro- and macroscopic aspects still remains imperfect. 
3.2. - LUDERS BANDS. Fig. 7 reproduces the typical yield behavior of a fine-grained polycrystalline mild steel at, or slightly above, room temperature. The initial yield drop, between an upper yield stress, $\sigma_{u}$, and a lower yield stress, $\sigma_{\ell}$, is followed by a plateau during which slip activity propagates through the specimen in form of one or several Lüders bands with a constant front velocity.

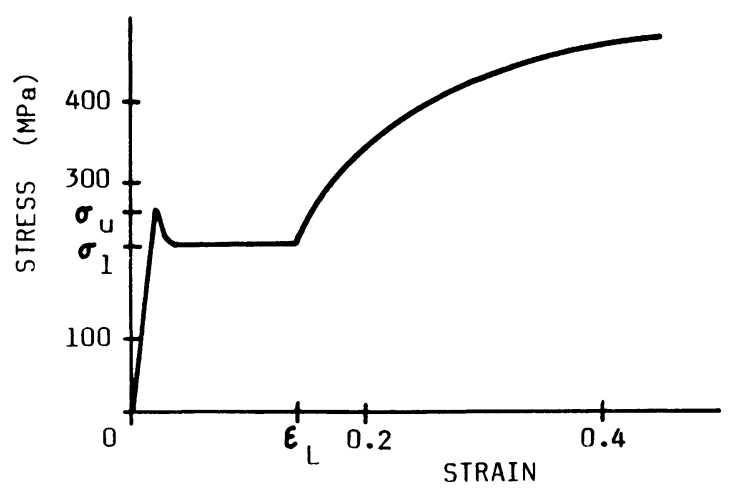

Fig. 7. - Typical stress-strain curve of a mild steel at room temperature. Lüders band propagation takes place at a constant stress, $\sigma_{\ell}$, and leaves the specimen in a uniform state of strain at $\varepsilon=$ $\varepsilon_{L}$.

When $\varepsilon=\varepsilon_{L}, \quad \varepsilon_{L}$ being the Lüders strain, typically a few percent, the front(s) have moved all along the specimen which is left in a uniform state of strain. Homogeneous deformation ensues.

The nucleation of Lüders bands is assisted by local stress concentrations, for instance at the grips, and several bands can be active simultaneously, originating from various places along the specimen. Yield behavior is not always as simple as pictured in fig. 7, but one usually refers to Lüders-like behavior whenever slip activity progresses through the specimen in an orderly manner. This is in particular the case for flat single crystals of f.c.c. alloys (4).

In phenomenological terms, the nucleation stress of the first band is larger than the stress required for further propagation. This implies that the SRS is not too large, so that the stress level effectively drops during the initial burst in strain rate. In microscopic terms, the equivalent formulation is that dislocations are initially aged by segregating solute atoms, so that the stress needed to liberate them is largely in excess of the glide resistance. The fast rate of liberation of mobile dislocations and their subsequent multiplication induce a large negative contribution to the SHR, according to eq. (11).

The evolution of strain rate, at a fixed position in the specimen, is as follows: there is an abrupt increase as slip activity starts, i.e. when the front crosses the considered section. With the local accumulation of strain, strain hardening, then, becomes positive and the strain rate decreases while the accumulated strain saturates to the value $\varepsilon_{L}$ (cf. $\S 2.3$ and figs. 5 and 6). Since the front propagates with a steady-state velocity, $v_{f},\left(v_{f} \simeq 1\right.$ $\mathrm{mm} . \mathrm{s}^{-1}$ ) this time evolution can be developed into an evolution in space (the strain rate is a function of the single evolutionary variable, $\left.x-v_{f} t\right)$. Thus, the space profile of the strain rate given in fig. 8 reflects the time profile of fig. 6. The strain rate increases rather abruptly to a peak value $\dot{\varepsilon}_{f}$, and gradually decreases. The Lüders band width, w. can be then defined as the thickness of the active region behind the front (cf. fig. 8).

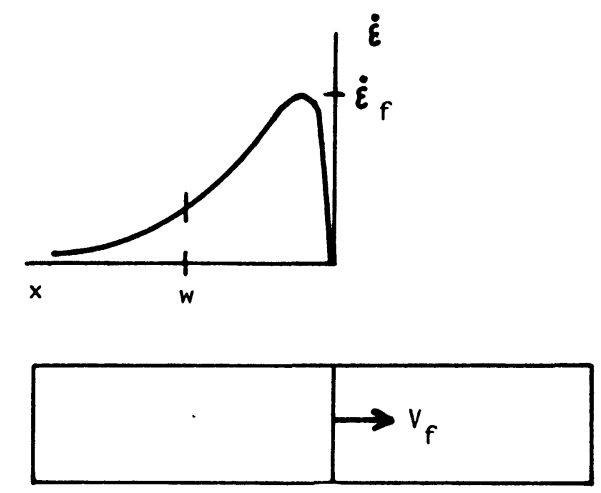

Fig. 8. - The strain rate profile of a Lüders band. $v_{f}, \dot{\varepsilon}_{f}$ and $w$ are the front velocity, the strain rate at the front and the Lüders band width, respectively. Actual band widths are smaller than suggested by this drawing $\left(w / L \simeq 10^{-2}\right)$.

In summary, we have clearly identified an instability of type $h$, with bounded amplitude. The spreading of slip is caused by the necessity for activating new sources in the specimen when old ones become strain-hardened. This qualitative description can be elaborated more formally at phenomenological level. Quite generally, it appears that the quantities to be estimated, $\sigma_{l}, v_{f}, w, \varepsilon_{L}$, and $\varepsilon_{F}$, cannot be unequivocally determined in terms of such material properties as the SRS, the alloy friction stress, some average strain hardening rate, or, better, the strain rate profile of the Lüders band, and the prescribed strain rate. Indeed, care must be taken when averaging material properties within a Lüders band, i.e. within a region where the strain rate and the local strain hardening rate are subject to large variations. The major difficulty, however, arises from the fact that one relation is missing between the investigated quantities (9).

To define more precisely the unknown term, we take the band width, w, as the typical length scale of the problem. Because of the steady state character of front propagation, $w=\delta t \cdot v_{f}$, where $\delta t$ is the corresponding time scale, i.e. the time interval during which there is a significant plastic activity in a given section. $\delta t$ can be evaluated by considering the dynamics of dislocation populations, as done in $\S 2$. An indeterminacy remains, however, if the front velocity is not specified. Two mechanisms are postulated to explain the orderly activation of slip, but the material or dislocation properties which control the occurrence or nonoccurrence of front propagation are still unclear. Locked dislocations ahead of the front can be freed under the action of internal stress peaks accompanying dynamic dislocation pile-ups. Alternatively the injection of cross-slipped dislocations into virgin regions can serve to initiate slip. In both cases it is expected that the front velocity should be directly related to the dislocation velocity at the front.

The same kind of indeterminacy is obtained with the PLC effect (33), and for the same reasons. To illustrate how the consideration of the evolution of dislocation populations in time and space may potentially solve this problem, we quote a result from the computer simulations of Lépinoux (20). 
Mobile screw dislocations are initially introduced within a slice of virgin crystal of thickness 0.5 $\mu \mathrm{m}$. They are allowed to move, multiply, annihilate and cross-slip according to rules which depend on an externally applied stress and on the sum of mutual elastic interactions up to large distances (equivalent to several $\mu \mathrm{m}$ ). In addition, they are subjected to a local strain hardening rate proportional to the accumulated strain. In fig. 9 is plotted the evolution with time of the strain rate profile in the simulated deforming specimen. The slip lamella expands with a constant front velocity, which is found to be proportional to the average dislocation velocity at the front(s). One can also notice in fig. 9 the decrease in slip activity behind the fronts. Thus, both $w$ and $v_{f}$ are determined. Further, it is found that no propagation at all is observed if the multiplication rate of dislocations falls beyond a critical value. The front velocity is found to be of the form:

$$
v_{f}=(1 / 2) v_{d}\left(1-\left(x / x_{c}\right)\right),
$$

where $v_{d}$ is the average dislocation velocity at the front; $X$ is a parameter governing the multiplication rate and $X$ the critical value such that no front propagation takes place when $x>x_{c}$, i.e. when the multiplication rate is too small. A similar result is obtained when simulating the dynamic behavior of initially aged edge dislocations.

In conclusion we see that a combination of elaborations at microscale and at mesoscale allows all length scales associated with the nonuniformity to be determined.

3.3. THE PORTEVIN-LE CHATELIER EFFECT. Jerky flow associated with the PLC effect is found in many substitutional or interstitial f.c.c. or b.c.c. dilute alloys. It is observed within a well-defined domain of temperature, approximately between 0.2 and $0.4 \mathrm{~T} / \mathrm{T}_{\mathrm{m}}$ : melting temperature). At a given temperature, it occurs between two critical values of the applied strain rate or stress rate.

Fig. 10 schematically reproduces the deformation curves obtained when an alloy is tested in the domain of jerky flow. Load drops and strain bursts are obtained in deformation with constant strain rate and constant stress rate, respectively. The two deformation curves of fig. 10 are, however, globally similar, as shown by Cuddy and Leslie for the case of a Fe-1.5\% Si alloy (34).

Each strain burst is associated with the steady state propagation of a deformation band, which is usually initiated at one grip and moves to the other end of the specimen with a constant front velocity $v_{f}, v_{f}$ is of the order of $10 \mathrm{~cm} \cdot \mathrm{s}^{-1}$, i.e. it is $\simeq 10^{2}$ times larger than the velocity of Lüders fronts. In constant strain rate tests, stress relaxation effects interfere with band propagation resulting in a complex PLC-band morphology. Fig. 11 shows an example of strain nonuniformities obtained in an Al-5\%Mg alloy tested at $300 \mathrm{~K}$ with a constant strain rate of $10^{-4} \mathrm{~s}^{-1}$ (35).
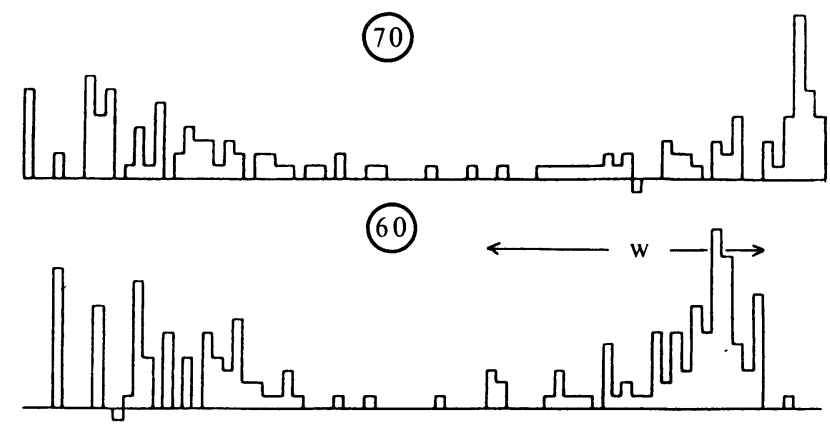

(50)

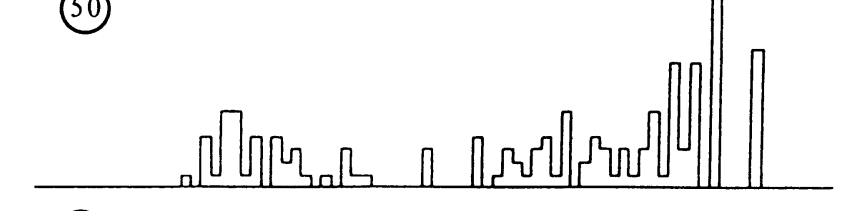

(40)

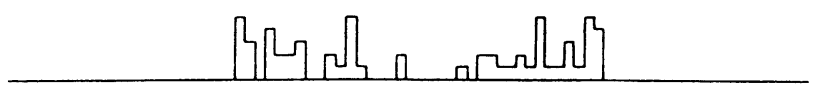

30
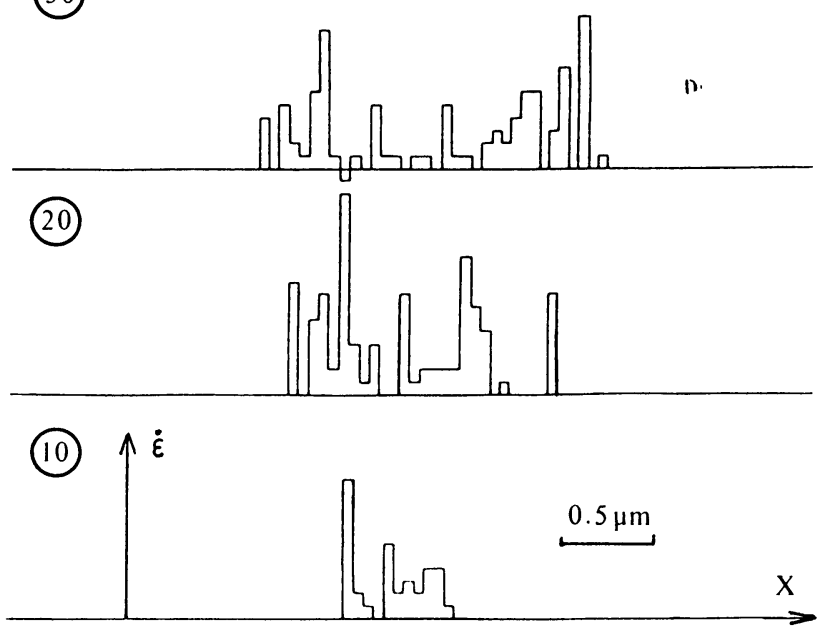

Fig. 9 - A simulation of Lüderslike slip propagation (see text for detail). The strain rate profiles along the specimen are indicated for several successive vaiues of time (encircled numbers) increasing from bottom to top. The initial configuration at $t=0$ consists of a slip lamella, of thickness $0.5 \mu \mathrm{m}$, containing mobile screw dislocations. The remaining parts of the specimen initially contain no dislocations.

Glide planes are vertical and the local strain rates are computed within each elementary slice as a function of time.

Slip propagates axially, by cross-slip, with a constant velocity and the strain rate profiles are similar to the one depicted in fig. 8.

After Lépinoux (unpublished work).

The microscopic mechanism causing the instability is dynamic strain aging (DSA),i.e. the segregation of solute atoms on the mobile dislocations. Since the 
early elaborations of Cottrell (36) and Friedel (25), many models have been proposed to account for the dynamic interaction of mobile dislocations with diffusing solute atoms. In most recent ones, the following view is preponderant (see e.g. (37, 38) and (9)). The solutes diffuse to dislocations arrested at localized obstacles to be overcome with the aid of thermal activation. The barrier height increases with time as solute concentration near the dislocation core continuously increases. It may happen, then, that deformation becomes "antithermally activated". The effective stress, $\sigma^{*}$, needed to overcome the barrier increases with the waiting time at the obstacle, $t_{w}$. Since $t_{w}$ is inversely proportional to the average dislocation velocity, $v$, we have $d \sigma^{*} / d \operatorname{lnv}<0$.

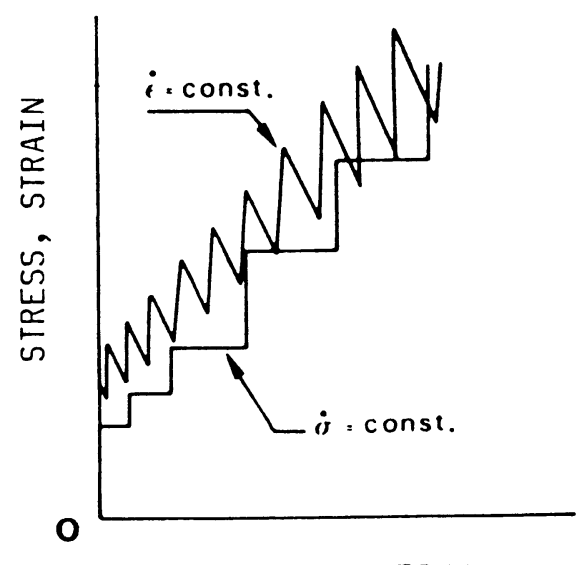

TIME

Fig. 10. - Typical shapes of deformation curves associated with the PLC effect at constant strain rate, $\sigma(t)$, or at constant stress rate, $\varepsilon(t)$.

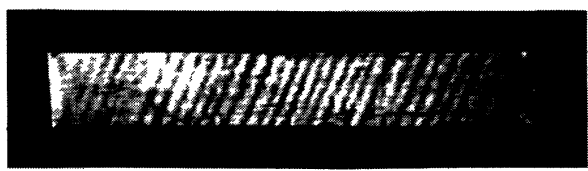

Fig. 11. - PLC bands in an Al-5\%Mg polycrystalline specimen tested at $300 \mathrm{~K}$ with a constant strain rate of $10^{-4} \mathrm{~s}^{-1}$. Specimen width is $5 \mathrm{~mm}$. After (35).

Going from microscale to mesoscale, we express the effective stress as $\sigma^{*}=\sigma-h \varepsilon$, where the SHR, $h$, is assumed to be constant around a fixed value of strain, and replace the average dislocation velocity by $\dot{\varepsilon} / \rho_{m}$ b, according to eq. (2). Even when taking into account the strain rate dependence of the mobile density, one expects to obtain a domain of experimental conditions where $\left(d \sigma^{*} / d \ln \dot{\varepsilon}\right)=$ $(\partial \sigma / \partial \ln \dot{\varepsilon})_{\varepsilon}$, i.e. the local SRS is negative (33, 39).

Thus, as shown in $\S 2.3$, we have an instability of type $S$ characterized by strain rate jumps. The behavior of the local strain rate in a specimen

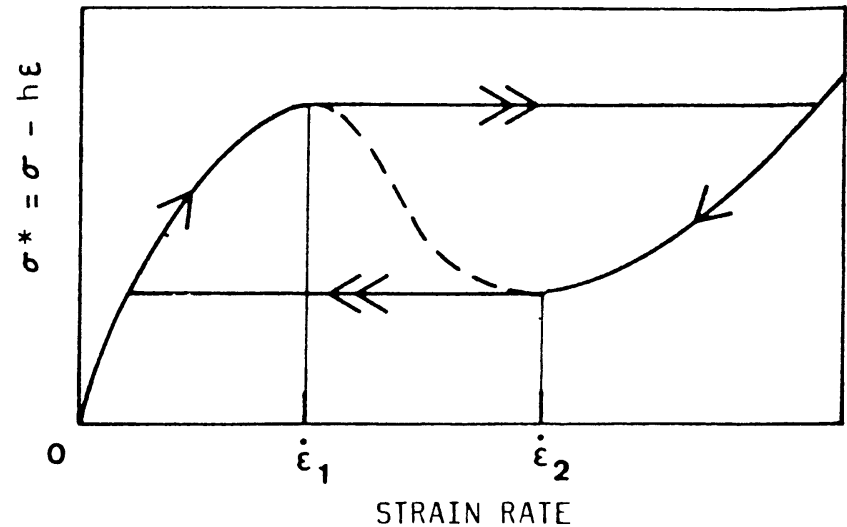

Fig. 12. - Relaxation oscillations associated with the PLC effect. The closed trajectory indicated by the arrows is periodically followed by the strain rate in a specimen cross-section. The double arrows denote the fast jumps occurring when the boundaries of the "forbidden gap", $\dot{\varepsilon}_{1}$ or $\dot{\varepsilon}_{2}$ are reached. After (33).

cross-section is illustrated in fig. 12 by the diagram of $\sigma^{*}$ vs $\dot{\varepsilon}$.

In the ascending branches of $\sigma^{*}(\dot{\varepsilon})$, the SRS is positive and uniform deformation is stable. At low strain rates (i.e. for large waiting times), dislocations drag their solute atmospheres and this requires relatively high stresses. At high strain rates (or small waiting times), dislocations move across a nearly immobile distribution of solute atoms, at comparatively low stresses. When the applied strain rate, $\dot{\varepsilon}_{a}$, is within the domain of negative SRS, which is bounded by two critical strain rates, $\dot{\varepsilon}_{1}$ and $\dot{\varepsilon}_{2}$, uniform deformation is unstable. During a deformation test, the strain rate first increases uniformly, until the value $\dot{\varepsilon}_{1}$ at which $S=0$ is reached. Then, the strain rate jumps at constant stress and strain (cf. fig. 12), to the branch of high $\dot{\varepsilon}$, which corresponds to the unlocking of dislocations from their solute atmospheres. Under the influence of strain hardening, the local strain rate then decreases, until the second critical value, $\dot{\varepsilon}_{2}$, is reached. A second jump in strain rate then occurs, which brings dislocations back to the branch of small strain rates where they are dynamically pinned by solute atoms dragged along.

In such conditions, the domain of strain rates bounded by the two critical values is forbidden to dislocations. When the applied strain rate lies within this "forbidden gap", local strain rates periodically describe a cycle with successive steps of fast-slow-fast-slow motion, with a time average equal to the prescribed value, $\dot{\varepsilon}_{a}$. This type of behavior is known as relaxation oscillations (40). At low temperatures this mechanism is not operative because solute diffusion is too slow. At high temperatures, the hardening effect of solute atoms vanishes. Therefore, the PLC effect can only occur within a well-defined temperature range.

The strain rate vs time profile in a given section of the specimen can be deduced from fig. 12. It is given in fig. 13, where one notices the gap in strain rates which results from relaxation oscillations. 


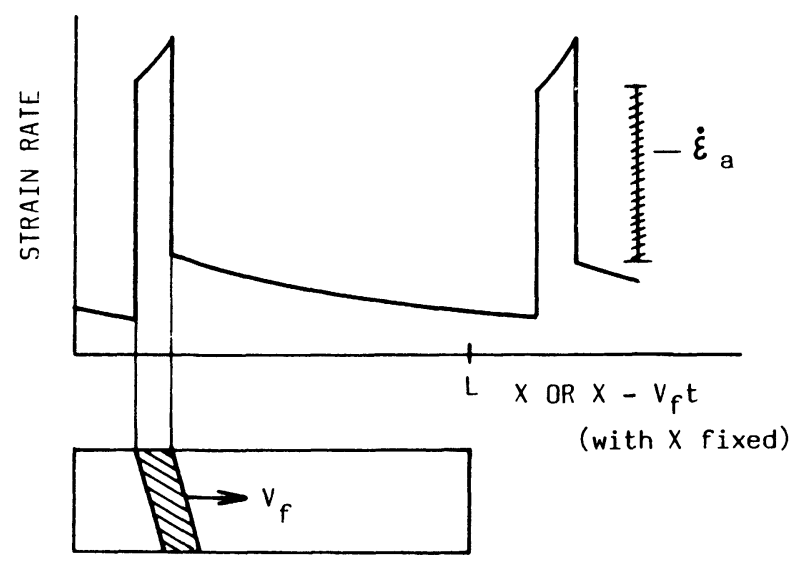

Fig. 13. - A PLC band and the corresponding strain rate profile, $\dot{\varepsilon}(x)$, which in conditions of steady state band propagation is identical to the time profile at a given location $x, \dot{\varepsilon}\left(x-v_{f} t\right)$.

As done in $\S 3.2$ for Lüders bands, one can develop the time profile into a spatial profile $\dot{\varepsilon}(x)$, since in conditions of constant stress rate testing PLC bands propagate with a constant velocity $v_{f}$. Again, there is only one evolutionary variable, $x-$ $v_{f} t$. This profile is represented in fig. 13, where one sees that the regions of high strain rates (typically $10^{2} \mathrm{~s}^{-1}$ ) correspond to the PLC band where dislocations are highly mobile. The band is bounded by two fronts where upward and downward strain rate jumps take place. In the remaining parts of the specimen, the background strain rates are much smaller, of the order of a typical creep rate.

Most probably, the dislocation mechanisms by which the upward strain rate jump propagates are similar to those discussed in $\S 3.2$, with regard to Lüders front propagation. The determination of the length and velocity scales associated with the PLC effect (band widths, front velocities) can then be performed in principle by considering in more detail the evolution in time and space of dislocation populations.

The mechanism of relaxation oscillations is very general. It is met whenever a relation between a flux and a force (or between a force and a flux) takes a sigmoïdal shape with a portion of negative slope. Several examples are listed and discussed in ref. (40), i.e., among others, "stick-slip" effects $(41,42)$, the Gunn effect in compound semiconductors, the pinning of Bloch walls by fiffusing carbon atoms in ferromagnetic Fe-Si specimens (43).

Although the connection between DSA and jerky flow associated with the PLC effect is now qualitatively understood, several problems remain unsolved. The details of the diffusion mechanisms which induce DSA, and, in particular their strain dependence are still under discussion (38). In many substitutional alloys, there is an "incubation" strain, $\varepsilon_{c}$, during which either deformation is uniform or Lüders band propagation takes place. Indeed, the occurrence of a Lüders band indicates that diffusion is ton slow to allow for dynamic strain aging. This is the reason why, in practice, many alloys exhibit Lüders-type instabilities at some temperature, and, further, the PLC effect at higher temperatures.

To explain the occurrence of this critical strain, it has been proposed that vacancy formation during plastic flow can enhance the diffusion of solutes in substitutional alloys. According to more recent investigations, the critical strain rather stems from the increase of the mobile dislocation density at the beginning of plastic flow. A simulation of unstable deformation curves by Neelakantan and Venkataraman (unpublished work) substantiates this view. The evolution with time of dislocation populations has been examined by those authors, using a simplified version of the considerations developed in $\S 2.2$, and with a sigmoïdal shape for the stress vs velocity law. The deformation curves thus obtained exhibit an incubation strain when suitable values for the multiplication rate and for the saturated density of mobile dislocations are introduced.

\section{Conclusion}

From this study of strain nonuniformities and plastic instabilities we draw the following conclusions. Plastic flow is inherently nonuniform and a coherent description of mechanical behavior involves simultaneous consideration of several scales (microscale, mesoscale, macroscule), at which instabilities can occur. Nonlinear cooperative processes are responsible for the occurrence of SNPI. They influence mechanical properties to the point that individual dislocation processes need not be described in full detail when a qualitative understanding is required.

The determination of length scales is a long standing problem in plasticity. The elaborations reported in this review are potentially well suited for dealing with this problem. Though not accomplished as yet, they have already yielded promising results.
(1) IOSS, G., JOSEPH, D.D., Elementary Stability and Bifurcation theory, (Springer-Verlag) 1980.

(2) NEUHAUSER, H., in Strength of Metals and alloys (ICSMA 5), eds. P. Haasen et al. (Pergamon (Press), vol. 3 (1980) 1531.

(3) NEUHAUSER, H., in Dislocations in Solids, ed. F.R.N. Nabarro (North-Holland), vol. 6 (1983) 321 .

(4) NEUHAUSER, H., in reference (7), below.

(5) ARLiON, A.S., in Inhomogeneity of Plastic Deformation, ed. R.E. Reed-Hill, (ASM) 1973.
(6) NICOLIS, G., PRIGOGINE, I., Self-0rganization in Nonequj.librium Systems ( $\mathrm{J}$. Wiley and Sons) 1977.

(7) Mechanical Properties and Behaviour of Solids Plastic Instabilities, eds. V. Balakrishnan and C.E. Bottani (World Scientific) 1986.

(8) Patterns, Defects and Microstructures in Nonequilibrium Systems, ed. D.Walnraef, NATO ASI series (Martinus Nijhoff) 1987.

(9) KOCKS, U.F., in Progress in Materials Science, Chalmers Anniversary Volume (Pergamon Press) 1981 , p. 185. 
(10) DEMIRSKI, V.V., KOMNIK, S.N., Acta Metall. 30 (1982) 2227.

(11) SCHWARZ, R.B., MITCHELL, J.W., Phys Rev. B9 (1974) 3292.

(12) Mechanical Behavior of Materials, eds. F. A. Mc Clintock and A.S. Argon (Addison Wesley) 1966 p. 295.

(13) BRECHET, Y., Doctorate Thesis, University of Grenoble (1987).

(14) ESTRIN, Y., KUBIN, L.P., Acta Metall. 34, (1986) 2455

(15) ANANTHAKRISHNA, G., SAHOO, D., J. Phys D: Appl. Phys. 14 (1981) 2081.

(16) ANANTHAKRISHNA, G., VASAKUMAR, M.C., J. Phys. D: Appl. Phys. 15 (1982) 2171.

(17) ANANTHAKRISHNA, G. Bull. Mater. Sci. 6 (1984) 665

(18) KOCKS, U.F., ARGON, A.S., ASHBY, M.F., Thermodynamics and Kinetics of Slip, Progress in Materials Science, eds. B. Chalmers et al (Pergamon Press) Vol 19 (1975) 89.

(19) Low Energy Dislocation Structures, eds. M.N. Bassim et al. (Elsevier Sequoia) 1986.

(20) LEPINOUX, J., KUBIN, L.P., Scripta Met. 21, (1987) 833.

(21) WALGRaEF, D., AIfantis, E.C., J. Appl. Phys. 58 (1985) 688, see also ref. (8) 257 and ref. (7) 314 and 354 .

(22) BOLLING, G.F., RICHMAN, R.H., Acta Metall. 13 (1965) 709 and 723.

(23) MUGHRABI, H., in Strength of Metals and Alloys (ICSMA 5), eds. P. Haasen, et al. (Pergamon Press) Vol. 3 (1979) 1615.

(24) NEUMANN, P., Acta Metall. 17 (1969) 1219, and ref. (8) 270.

(25) FRIEDEL, J., Dislocations (Pergamon Press) 1967.
(26) STRUDEL, J.L., in Dislocations et Déformation Plastique, eds. P. Groh et al. (Les Editions de Physique) 1979.

(27) HAASEN, P., in Physical Metallurgy, ed. R.W. Cahn (North-Holland) 1970 ,

(28) PIOBERT, G., (1836), cf. Mémorial Artillerie V (1842) 525.

(29) LUDERS, W., Dinglers Polytechnisches J. 156 (1860) 18.

(30) LE CHATELIER, F., Rev. de Métallurgie 6 (1909) 914.

(31) PORTEVIN, A., LE CHATELIER, F., Trans. ASST 5 (1924) 457

(32) HALL, E.D., Yield Point Phenomena in Metals and alloys (Mc Millan) 1970.

(33) KUBIN, L.P., ESTRIN, Y., Acta Metall. 33 (1985)

(34) 97.ंDY, L.J., LESLIE, w.C., Acta Metall. 20 (1972) 1157

(35) CHIHAB, K., ESTRIN, Y., KUBIN, L.P., VERGNOL, J., Scripta Met. 21 (1987) 203.

(36) COTTRELL, A.H., Dislocations and Plastic Flow in Solids (Clarendon Press) 1953.

(37) Van den BEUKEL, A., Phys. Stat. Sol. (a) 30 (1975) 197.

(38) Van den BEUKEL, A., KOCKS, U.F., Acta Metall. 30 (1982) 1027.

(39) PENNING, P., Acta Metall. 20 (1972) 1169.

(40) KUBIN, L.P., ESTRIN, Y., J. de Physique 47 (1986) 497.

(41) MAUGIS, D., BARQUINS, M., J. Phys. D: Appl. Phys. 11 (1978) 1979.

(42) MAUGis, D., J. Mat. Sci. 20 (1985) 3041.

(43) MARTIN, N., GLANGEAUD, F., GUILLET, D., PORTESEIL, J.L., J. Phys. C:Solid State Phys. 19 (1986) 407. 